\title{
The Need for Polarization for Extracting Baryon Resonances and the NSTAR Program at CLAS
}

\author{
Philip L. Cole (for the CLAS Collaboration) \\ Idaho State University, Dept. of Physics, Pocatello, Idaho 83209 USA cole@physics.isu.edu
}

\begin{abstract}
We report on the NSTAR program in Hall B of JLab on using polarization observables to extract parameters of baryon resonances. The scientific purpose of the program is to improve the understanding of the underlying quark degrees of freedom, especially in the higher resonance regions, where we expect to uncover many of missing baryon resonances that mainly decay through multi-meson channels. With the high-quality beam of circularly- and linearly-polarized photons onto unpolarized and polarized proton and deteurium targets, and coupled with the nearly complete solid angle coverage of CLAS, we will extract the differential cross sections and associated polarization observables obtained by the photoproduction of vector mesons and kaons at center of mass energies of 1.7 to $2.2 \mathrm{GeV}$. The paper will primarily present the photon beam aspects of the excited baryon program.
\end{abstract}

Keywords: Photoproduction, Polarization, Baryon Spectroscopy

PACS: $13.60 . \mathrm{Le}, 13.60 . \mathrm{Rj}, 25.20 . \mathrm{Lj}, 29.25 . \mathrm{Pj}, 29.27 . \mathrm{Hj}$

\section{INTRODUCTION}

A fundamental scientific goal for Jefferson Lab is to understand the structure and interactions of protons, neutrons, and other hadrons. The nature of the ground state and excited states of the nucleon is sensitive to the details of the quark and gluon interactions. Data on the existence, masses, and partial widths of the baryons arising from the excited spectrum of underlying quarks are therefore necessary for fully understanding the fundamental model of the strong force, QCD. The excited baryon program at JLab $[1,2]$ has two primary objectives: (a) probe the transition form factors as functions of $Q^{2}$ of the known resonances to gain insight into their internal structure and hence the confining potential for this 3-quark system, and (b) systematically study the spectroscopy of the excited baryon states that decay through $N \pi, N \eta, N \pi \pi, N \omega, N \rho$, and $K Y$.

An outstanding problem in our current day understanding of baryon spectroscopy is the conundrum of the missing resonances. $\mathrm{SU}(6) \otimes \mathrm{O}(3)$ symmetric quark models predict far more resonances than have thus far been observed. Most of our knowledge of the baryon resonance spectrum has come from the $\pi N \rightarrow \pi N$ analyses and is applicable only to resonances with significant $N \pi$ coupling [3]. One solution is to restrict the number of internal degrees of freedom by demanding that two quarks be bound into a diquark pair [4] and thereby lowering the overall level density of baryon resonances. An alternate three-constuent-quark solution was put forward by Koniuk and Isgur [5] and others [6,7]. A nice review article on several models of excited baryon spectra and corresponding strong decay channels can found in Ref. [8].

CP947, VII Latin American Symposium on Nuclear Physics and Applications edited by R. Alarcon, P. L. Cole, C. Djalali, and F. Umeres

(C) 2007 American Institute of Physics 978-0-7354-0461-8/07/\$23.00 
At center-of-mass energies below $1.7 \mathrm{GeV}$, the single pion production channel dominates both the pion and photoabsorption cross sections. As the c.m. energy increases towards $2.0 \mathrm{GeV}$ the two- and three-pion decay channels become more dominant, and it is in this important energy region that the masses and partial widths of the resonances are poorly wdetermined. In these various calculations it has been found that the missing resonances tend to couple weakly to the $\pi N$ channel but stronger to the $\rho N, \pi \Delta$, and $\omega N$ channels. Quark models, moreover, predict existences of excited baryons, along with their masses, widths, spin/parity, and the associated angular distributions of the decay mesons. In Table 1 we tabulate a subset of the $\mathrm{SU}(6) \otimes \mathrm{O}(3)$ supermultiplet assignments.

\section{THE NEED FOR POLARIZATION OBSERVABLES}

Although there have been remarkable advances in electromagnetic meson production [ 9 , $10]$ and great strides in understanding the transition from ground state $\left[56,0^{+}\right]$to the $\left[70,1^{-}\right]$supermultiplet [11] over the past decade, there still remains a dire need for high-quality data with polarized targets and beams. Unpolarized cross sections only give information on the sum of the modulus squared of the amplitudes and are not sensitive to the cross or interference terms. Polarization observables give information on phases as well as setting conditions on the bilinear combinations of the amplitudes with all attendant algebraic constraints and inequalities. Spin observables therefore provide an incisive tool for distinguishing among the various quark models for the presence or absence of baryon resonances, be they missing, well established, and/or contentious, such as the $P_{11}(1710)[12,13,14]$. The following discussion is meant as a motivation for why polarization observables are crucial; it is by no means an exhaustive treatment, but reflects, rather, some highlights. See also Ref. [15] in these proceedings.

$$
\gamma p \rightarrow \omega p
$$

Without polarization constraints, it is not possible to readily disentangle the relative contributions of the many known baryon resonances, not even taking into account the conjectured missing ones. The photoproduction of the omega meson is a case in point [16]. Various models incorporate various resonances. Below we cite just three.

- Z.P. Li and Q. Zhao $[17,18]$ predict considerable contributions to $\omega N$ from the subthreshold states $S_{11}(1535)$ and $D_{13}(1520)$. Near threshold, the dominant contributions arise from the $F_{15}(1680)$ and the $P_{13}(1720)$. The effects from the twostar resonances $P_{13}(1900)$ and $F_{15}(2000)$ are relatively suppressed, but contribute nonetheless. At slightly higher energies, the $F_{15}(2000)$ plays an increasing role and - slightly suppressed - from $P_{13}(1900)$.

- Y. Oh, A.I. Titov, and T-S.H. Lee [19] explicitly include baryon resonances by incorporating Breit-Wigner descriptions and vertex functions provided by the relativized quark model of Capstick and Roberts.[6], where the dominant contributions arise from the missing $P_{13}(1910)$ as well as the $D_{13}(1960)$ - which is identified with the PDG $D_{13}(2080)$ - and the $G_{17}(2190)$, with lesser contributions from the $F_{15}(2000)$. 
TABLE 1. SU(6) $\otimes O(3)$ supermultiplet assignments from the QCD-improved model of Cutkosky for the measured and missing baryon resonances in the $N=2$ regime. The boxed supermultiplets are fully consistent with the diquark model. Missing resonances are not assigned a star rating.

\begin{tabular}{|c|c|c|c|c|c|}
\hline $\mathrm{N}^{*}$ & Status & $\mathrm{SU}(6) \otimes \mathrm{O}(3)$ & $\Delta^{*}$ & Status & $\mathrm{SU}(6) \otimes \mathrm{O}(3$ \\
\hline$D_{13}(1520)$ & $* * * *$ & $\left(56,0^{+}\right)$ & $P_{31}(1910)$ & $* * * * *$ & $\left(56,2^{+}\right)$ \\
\hline $\begin{array}{l}P_{11}(1710) \\
P_{11}(1880) \\
P_{11}(1975)\end{array}$ & $* * * *$ & $\begin{array}{l}\left(70,0^{+}\right) \\
\left(70,2^{+}\right) \\
\left(20,1^{+}\right)\end{array}$ & $P_{31}(1835)$ & & $\left(70,0^{+}\right)$ \\
\hline$P_{13}(1720)$ & $* * * * *$ & $\left(56,2^{+}\right)$ & $P_{33}(1600)$ & $* * *$ & $\left(56,0^{+}\right)$ \\
\hline $\begin{array}{l}P_{13}(1900) \\
P_{13}(1910) \\
P_{13}(1950) \\
P_{13}(2030)\end{array}$ & $* *$ & $\begin{array}{l}\left(70,2^{+}\right) \\
\left(70,2^{+}\right) \\
\left(70,2^{+}\right) \\
\left(20,1^{+}\right) \\
\end{array}$ & $\begin{array}{l}P_{33}(1920) \\
P_{33}(1985)\end{array}$ & $* * * *$ & $\frac{\left(56,2^{+}\right)}{\left(70,2^{+}\right)}$ \\
\hline$F_{15}(1680)$ & $* * * * *$ & $\left(56,2^{+}\right)$ & $F_{35}(1905)$ & $* * * * *$ & $\left(56,2^{+}\right)$ \\
\hline $\begin{array}{l}F_{15}(2000) \\
F_{15}(1995)\end{array}$ & $* *$ & $\begin{array}{l}\left(70,2^{+}\right) \\
\left(70,2^{+}\right)\end{array}$ & $F_{35}(2000)$ & $* *$ & $\left(70,2^{+}\right)$ \\
\hline$F_{17}(1990)$ & $* *$ & $\left(70,2^{+}\right)$ & $F_{37}(1950)$ & $* * * * *$ & $\left(56,2^{+}\right)$ \\
\hline
\end{tabular}

- The Giessen K-matrix coupled-channel approach of G. Penner and U. Mosel [20] has the $P_{11}(1710)$ dominating at threshold, with reduced importance of other resonances. Only the $J^{P}=\frac{3}{2}^{+}$contributions of the $P_{13}(1720)$ and the $P_{13}(1900)$ remain non-negligible.

\section{Beam Helicity Asymmetries for $\vec{\gamma} p \rightarrow p \pi^{+} \pi^{-}$}

A recent analysis of the $\gamma p \rightarrow p \pi^{+} \pi^{-}$channel by S. Strauch [21] extracted the beam-helicity asymmetries from circularly-polarized photons onto an unpolarized target with the CEBAF Large Acceptance Spectrometer (CLAS) [22]. The center of mass energy ranges between 1.35 and $2.30 \mathrm{GeV}$ and this analysis marks the first time such a study with circularly-polarized photons has been conducted in the resonance region. As evidenced in Fig. 1 the large cross-sectional asymmetries exhibit strong sensitivity to the dynamics of the reaction, and as shown in the superimposed fits, current models do not adequately describe the data over the entire kinematic range.

$$
\gamma N \rightarrow K \Lambda
$$

A recent experiment from CLAS [25] extracted the beam-recoil observables $C_{x}$ and $C_{z}$ in hyperon production using circularly-polarized photons onto unpolarized protons. These data are reproduced in Figs. 4 and 5 in Ref. [15] of these proceedings. What we see is the astonishing result the $\Lambda$ polarization is constistent with unity for all values of 


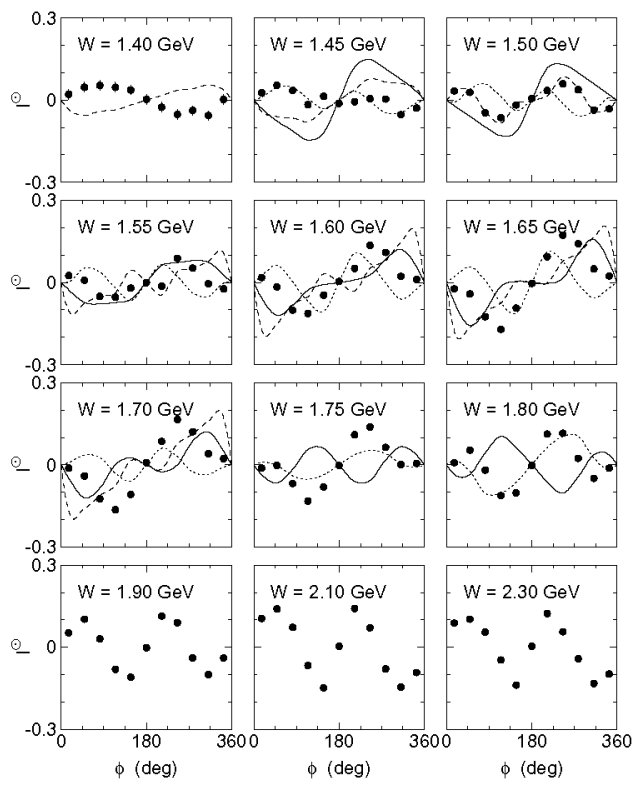

FIGURE 1. Azimuthal angular distributions for several c.m. energy bins of the cross-section asymmetry for $\vec{\gamma} p \rightarrow p \pi^{+} \pi^{-}$[21]. The solid and dotted curves are by Mokeev al. [23] and the dashed curves are results from Fix and Arenhövel [24].

c.m. energy and production angles. R. Schumacher [26] puts forward the argument that the photon fluctuates into a $\phi$ meson and by means of VDM carries all the spin of the photon. From this $s \bar{s}$ state, the $s$ quark combines with the $u$ and $d$ quark pair within the proton to form the $\Lambda$ baryon; the spinless $K^{+}$meson results from the remaining $\bar{s}$ and $u$ quarks. In this picture the $s$ quark remains completely polarized thereby polarizing the $\Lambda 100 \%$. The authors in Ref. [27], furthermore, argue that these newly-measured spin coefficients, $C_{x}$ and $C_{z}$, lend further evidence for the existence of the two-star $P_{13}(1900)$ resonance, which is outside the predicted states for diquark-quark models (cf. Table 1).

Hyperon production off deuterium with linearly-polarized photons forms the focus of the $\mathrm{g} 13$ experiment and is described in detail in Refs. [28, 29]. Preliminary results from $\vec{\gamma} p \rightarrow K \Lambda$ from the g8a run [30] indicate that polarized observables serve to delineate the relative contributions from the various baryon resonances. The plot in Fig. 2 shows the extracted photon beam asymmetry for four bins in $\theta_{c m}\left(K^{+}\right)$, where we superimpose quark model calculations by Stijn Janssen et al. [31, 32]. This calculation begins with Born terms and a core set of baryon resonances (see Ref. [32] for more details). Other curves are obtained by adding or removing selected resonant states that have been identified as possibly contributing to the differential cross section of the $K^{+} \Lambda$ channel. The positive photon asymmetry in the measurable angular range tends to favor the contribution of the $D_{13}(1895)$ (also known as the $D_{13}(1960)$ missing resonance). This controversial resonance was first predicted in the relativized pair-creation constituent quark model of Capstick and Roberts [33], where four states of mass around $1900 \mathrm{MeV}$ 
TABLE 2. CLAS experiments: search for excited baryon states.

\begin{tabular}{||l|c|ccc|c||}
\hline \hline Experiment & Reactions & Beam Pol. & Tgt Pol. & Recoil & Status \\
\hline \hline $\mathrm{g} 1 / \mathrm{g} 10$ & $\gamma p \rightarrow N \pi, p \eta, N \pi \pi, K \Lambda / \Sigma$ & - & - & $\Lambda, \Sigma$ & completed \\
$\mathrm{g} 8 \mathrm{a} / \mathrm{b}$ & $\vec{\gamma} p \rightarrow p(\rho, \omega, \phi, \eta), N \pi \pi, K \Lambda / \Sigma$ & linear & - & $\Lambda, \Sigma$ & completed \\
$\mathrm{g} 13$ & $\vec{\gamma} d \rightarrow p(\rho, \omega, \phi, \eta), N \pi \pi, K \Lambda / \Sigma$ & linear & - & $\Lambda, \Sigma$ & completed \\
\hline \hline $\mathrm{g} 9-\mathrm{gROST}$ & $\vec{\gamma} \vec{p} \rightarrow p(\rho, \omega, \phi, \eta), N \pi \pi, K \Lambda / \Sigma$ & lin/circ. & long/trans & $\Lambda, \Sigma$ & $2007 / 2008$ \\
$\mathrm{~g} 14-\mathrm{HD}$ & $\vec{\gamma} \vec{d} \rightarrow N(\rho, \omega, \phi, \eta), N \pi \pi, K \Lambda / \Sigma$ & lin/circ. & long/trans & $\Lambda, \Sigma$ & $2009 / 2010$ \\
\hline
\end{tabular}

have a significant $K \Lambda$ decay width [34]. A resonance-like structure in the cross section for $p\left(\gamma, K^{+}\right) \Lambda$ was observed in the 1998 SAPHIR data [35] and Mart and Bennhold [36] took this structure as evidence for the $D_{13}(1895)$ resonance. Linear polarization data will help serve to resolve the matter of whether the $D_{13}(1895)$ resonance exists or not.



Thu Aug 26 13:53:43 2004

FIGURE 2. Preliminary photon beam asymmetry of $\vec{\gamma} p \rightarrow K^{+} \Lambda$ compared with model calculations [31] including a set of possible 'missing' resonances.

\section{EXPERIMENTS}

What characteristics should any experiment have to provide the best constraints for coupled-channels calculations? And the answer is threefold: the set of experiments must possess wide kinematic coverage, have small systematic and statistical uncertainties, and finally allow for the extraction of several polarization observables. The high acceptance of the CEBAF Large Acceptance Spectrometer [22] coupled with polarized beams and polarized targets presents a wide range of options for extracting polarization observables as summarized in Table 2.

The linearly-polarized photons are produced from the coherent bremstrahlung facility $[37,38]$, which was commissioned during the g8 running period [30]. The details of 
TABLE 3. CLAS experiments: polarization observables in pseudoscalar photoproduction, i.e. $\gamma p \rightarrow K N$. See Ref. [42].

\begin{tabular}{||c|c|c|c|c||}
\hline \hline Observable & $\gamma$ & proton & Hyperon & polarization \\
\hline$\sigma_{0}$ & - & - & - & unpolarized \\
\hline$P$ & - & - & $y^{\prime}$ & Single \\
$\Sigma$ & $\operatorname{lin} .-\pi / 2$ & - & - & \\
$T$ & - & $y$ & - & \\
\hline \hline$+E$ & circ. & $z$ & - & Beam-Target \\
$+F$ & circ & $x$ & - & \\
$+G$ & $\operatorname{lin} .-\pi / 4$ & $z$ & - & \\
$-H$ & $\operatorname{lin} .-\pi / 4$ & $x$ & - & \\
\hline \hline$-C_{x}$ & $\operatorname{circ}$ & - & $x^{\prime}$ & Beam-Recoil \\
$+C_{z}$ & $\operatorname{circ}$ & - & $z^{\prime}$ & \\
$+O_{x}$ & $\operatorname{lin} .-\pi / 4$ & - & $x^{\prime}$ & \\
$+O_{z}$ & $\operatorname{lin} .-\pi / 4$ & - & $z^{\prime}$ & \\
\hline$+T_{x}$ & - & $x$ & $x^{\prime}$ & Target-Recoil \\
$+T_{z}$ & - & $x$ & $z^{\prime}$ & \\
$-L_{x}$ & - & $z$ & $x^{\prime}$ & \\
$+L_{z}$ & - & $z$ & $z^{\prime}$ & \\
\hline
\end{tabular}

the Frost target [39] and the ensuing double-polarization program is discussed in detail in Refs. $[15,40]$ in these proceedings. The HD target [41] will provide logitudinally polarized neutrons. Both the FROST and HD program will offer a suite of experiments detecting pseudoscalar mesons produced with beams of linearly- and circularly-polarized photons. The polarization observables obtained through the experiments, g1/g10, g8a/b, $\mathrm{g} 9, \mathrm{~g} 13, \& \mathrm{~g} 14$, will provide rigid constraints on partial-wave and/or coupled-channel analysis. See Table 3 and Ref. [42]. Note that the quantization axes are defined as: $\mathbf{z}=\hat{\mathbf{p}}_{p}$, $\hat{\mathbf{y}}=\left(\mathbf{p}_{\gamma} \times \mathbf{p}_{K}\right) /\left|\mathbf{p}_{\gamma} \times \mathbf{p}_{K}\right|, \hat{\mathbf{x}}=\hat{\mathbf{y}} \times \hat{\mathbf{z}}, \hat{\mathbf{z}}^{\prime}=\hat{\mathbf{p}}_{\Lambda}, \hat{\mathbf{y}}^{\prime}=\hat{\mathbf{y}}$, and $\hat{\mathbf{x}}^{\prime}=\hat{\mathbf{y}}^{\prime} \times \hat{\mathbf{z}}^{\prime}$. The following algebraic relations will serve to further constrain the analyses and provide a check on the systematics:

- $F G-E H=P-T \Sigma$

- $T_{x} L_{z}-T_{z} L_{x}=\Sigma-P T$

- $C_{z} O_{x}-C_{x} O_{z}=T-P \Sigma$

- $T_{x}^{2}+T_{z}^{2}+L_{x}^{2}+L_{z}^{2}=1-T^{2}+\Sigma^{2}+P^{2}$

- $C_{x}^{2}+C_{z}^{2}+O_{x}^{2}+O_{z}^{2}=1+T^{2}-\Sigma^{2}-P^{2}$

$\cdot E^{2}+F^{2}+G^{2}+H^{2}=1-T^{2}-\Sigma^{2}-P^{2}$

\section{ANALYSIS AND THEORETICAL ADVANCES}

The double and triple polarized observables arising from photoproduction of vector mesons and hyperons demand thorough analysis. There are several ways to extract these 
observables:

- spin density matrix elements. See: Refs. [43, 38, 44]

- helicity and hybrid helicity-transversity basis. See Ref. [45].

- asymmetry parameters to extract the spin polarization observables in the photonnucleon c.m. frame as delineated in Table 3.

No matter which basis one chooses, one can go from one to another, as described in Ref. [46] on transforming from spin density matrix elements to spin observables in the photon-nucleon c.m. frame. Perhaps analyzing the data in different bases will further serve to understand the systematics.

To strengthen the efforts in analyzing this forthcoming torrent of data, the Excited Baryon Analysis Center (EBAC) was established at JLab in January of 2006. A dynamical coupled-channel analysis of the data for photo and electro-production of $\pi, \eta, \omega, K$, and $\pi \pi$ is underway at EBAC. Indeed the goal of EBAC is to reach the DOE milestone by 2009: "Complete the combined analysis of available pion, eta, and kaon photoproduction data for mucleon resonances and incorporate analysis of two-pion final states into the coupled channel analysis of resonances" [47]. And experimentally identifying these baryon resonances through a robust coupled-channel analysis will allow for the comparison of fundamental predictions from Lattice QCD $[48,49]$ for the masses and structure of these states.

\section{OUTLOOK}

We are living in exciting times. We are poised on the threshold of understanding how the internal quark symmetries make up the underlying structure of baryon resonances. The observables and asymmetries afforded by polarized beams and polarized targets provide a powerful means for measuring the properties of excited states of nucleons, which ultimately will give us insight into how quarks are confined.

\section{ACKNOWLEDGMENTS}

This work was made possible through a grant from the National Science Foundation, NSF-0555497. The author also gratefully acknowledges NSF Award 0652360, procured through the Office of International Science and Engineering. This grant helped sponsor sixteen students, who traveled from the U.S. to Cusco, Peru to attend the VII Latin American Symposium for Nuclear Physics and Applications. All the sponsored students gave talks at the conference and also contributed to these proceedings. Jefferson Science Associates operates the Thomas Jefferson National Accelerator Facility under DOE contract DE-AC05-06OR23177.

\section{REFERENCES}

1. V. Burkert, R. Gothe, T.S.H. Lee et al., "Excited Baryon Program at JLab," Contribution to the NSAC Long Range Plan, Jan. 11, 2007. 
2. Whitepaper. DNP Town Meeting on Hadronic Physics, X. Ji \& Z.-E. Meziani, co-chairs, April 2007.

3. R.L. Workman, Review of Particle Physics, Journ. Phys. G 33 (2006) 969 and references therein.

4. D.B. Lichtenberg, Phys. Rev. 178 (1969) 2197;

R.E. Cutkosky and R.E. Hendrick, Phys. Rev. D 16 (1977) 2902.

5. R. Koniuk and N. Isgur, Phys. Rev. D 21 (1980) 1868.

6. S. Capstick and W. Roberts, Phys. Rev. D 47 (1993) 1994;

7. S. Capstick and B.D. Keister, Phys. Rev. D 51 (1995) 3598.

8. S. Capstick and W. Roberts, Prog. Part. and Nucl, Phys. 45 (2000) 241.

9. V.D. Burkert and T.-S.H. Lee, Int. J. Mod. Phys. E 13 (2004) 1035. [arXiv:nucl-ex/0407020].

10. T.-S.H. Lee and L.C. Smith, arXiv:nucl-th/0611034v1, 10 Nov. 2006. submitted to J. Phys. G (2006).

11. V.D. Burkert, R. De Vita, M. Battaglieri, M. Ripani, and V. Mokeev, Phys. Rev. C 67 (2003) 035204.

12. I. Aznauryan et al. Phys. Rev. C 72 (2005) 045201.

13. T. Mart and A. Sulaksono, Phys. Rev. C 74 (2006) 055203.

14. R. Arndt, W. Briscoe, I. Strokovsky, and R. Workman, Phys. Rev. C 74 (2006) 045205.

15. V. Crede, contribution to these proceedings.

16. F.J. Klein and P.L. Cole, NSTAR2004 Proceedings p. 146. (World Scientific) ISBN 981-256-090-4.

17. Q. Zhao, Z.P. Li, C. Bennhold, Phys. Lett. B 436 (1998) 42; Phys. Rev. C 58 (1998) 2393.

18. Q. Zhao, Phys. Rev. C 63 (2001) 025203.

19. Y. Oh, A. Titov, T.-S.H. Lee, Phys. Rev. C 63 (2001) 025201;

Y. Oh and T.-S.H. Lee, Phys. Rev. C 66 (2002) 045201.

20. G. Penner and U. Mosel, Phys. Rev. C 66 (2002) 055212.

21. S. Strauch et al. (CLAS Collaboration), Phys. Rev. Lett. 95 (2005) 162003.

22. B.A. Mecking et al., Nucl. Instrum. and Methods A 503/3 (2003) 513.

23. V.I. Mokeev et al., Yad. Fiz 64 (2001) 1368;

NSTAR2004 Proceedings p. 321 (World Scientific) ISBN 981-256-090-4.

24. A. Fix and H. Arenhövel, Eur Phys. J. A25 (2005) 115.

25. R. Bradford et al. (CLAS Collaboration) Phys. Rev. C 75 (2007) 035205.

26. R. Schumacher, arXiv:nucl-ex/06112035. To be published in Eur. Phys. J. A.

27. V.A. Nikonov, A.V. Anisovich, E. Klempt, A.V. Sarentsev, and U. Thoma, ArXiv:hep-ph/0707.3600v1.24 July 2007.

28. E. Munevar, contribution to these proceedings.

29. The run period g13 is formed of the experiments: E06-103 and a CLAS Approved Analysis on deuteron disintegration asymmetries.

30. The run period g8 is formed of the experiments: E94-109, E98-109, E99-013, and two CLAS Approved Analyses for the $K \Lambda$ and $p \eta, \eta^{\prime}$ channels.

31. S. Janssen, J. Ryckebusch, D. Debruyne, and T. Van Cauteren, Phys. Rev. C 65 (2001) 015201.

32. S. Janssen, D.G. Ireland, and J. Ryckebusch, Phys. Lett. B 562 (2003) 51.

33. S. Capstick and W. Roberts, Phys. Rev. D 49 (1994) 4570.

34. S. Capstick and W. Roberts, Phys. Rev. D 58 (1998) 074011.

35. SAPHIR Collaboration: M.Q. Tran et al., Phys. Lett. B 445 (1998) 20.

36. T. Mart and C. Bennhold, Phys. Rev. C 61 (2000) 012201.

37. P.L. Cole, J. Kellie, F.J. Klein, K. Livingston, J.A. Mueller, J.C. Sanabria, and D.J. Tedeschi, EMI2001, (World Scientific) ISBN 981-238-044-2. (2002).

38. J. Salamanca, contribution to these proceedings

39. The run period $\mathrm{g} 9$ is formed of the experiments: E02-112, E03-105, E04-102, E05-012, E06-013.

40. C. Hanretty, contribution to these proceedings.

41. The run period g14 is formed of the experiment: E06-101 (so far).

42. R.A. Adelseck and B. Saghai, Phys. Rev. C 42 (1990) 108.

43. K. Schilling, P. Seyboth, and G. Wolf, Nucl. Phys. B 15 (1970) 397.

44. Qiang Zhao, J.S. Al-Khalili, and P.L. Cole, Phys. Rev. C 71 (2005) 054004.

45. W. Roberts and T. Oed, Phys. Rev. C 71 (2005) 055201.

46. W.M. Kloet and Frank Tabakin, Phys. Rev. C 61 (1999) 015501.

47. Excited Baryon Analysis Center: http://ebac-theory.jlab.org/

48. A. Lichtl, contribution to these proceedings; arXiv:hep-lat/0708.2072v2. 16 Aug 2007.

49. J. Bulava, contribution to these proceedings. 